\title{
Robots Providing Cognitive Assistance in Shared Workspaces
}

\author{
Paul Baxter \\ L-CAS, School of Computer Science \\ University of Lincoln, U.K. \\ pbaxter@lincoln.ac.uk
}

\author{
Peter Lightbody \\ L-CAS, School of Computer Science \\ University of Lincoln, U.K. \\ plightbody@lincoln.ac.uk
}

\author{
Marc Hanheide \\ L-CAS, School of Computer Science \\ University of Lincoln, U.K. \\ mhanheide@lincoln.ac.uk
}

\begin{abstract}
Human-Robot Collaboration is an area of particular current interest, with the attempt to make robots more generally useful in contexts where they work side-by-side with humans. Currently, efforts typically focus on the sensory and motor aspects of the task on the part of the robot to enable them to function safely and effectively given an assigned task. In the present contribution, we rather focus on the cognitive faculties of the human worker by attempting to incorporate known (from psychology) properties of human cognition. In a proof-of-concept study, we demonstrate how applying characteristics of human categorical perception to the type of robot assistance impacts on task performance and experience of the participants. This lays the foundation for further developments in cognitive assistance and collaboration in side-by-side working for humans and robots.
\end{abstract}

\section{CCS CONCEPTS}

- Human-centered computing $\rightarrow$ Laboratory experiments; $\bullet$ Computing methodologies $\rightarrow$ Cognitive robotics;

\section{KEYWORDS}

Cognitive Assistance; Cognitive Collaboration; Shared Workspace

\section{ACM Reference Format:}

Paul Baxter, Peter Lightbody, and Marc Hanheide. 2018. Robots Providing Cognitive Assistance in Shared Workspaces. In HRI '18 Companion: 2018 ACM/IEEE International Conference on Human-Robot Interaction Companion, March 5-8, 2018, Chicago, IL, USA. ACM, New York, NY, USA, Article 4, 2 pages. https://doi.org/10.1145/3173386.3177070

\section{INTRODUCTION}

In this work, the focus is on the cognitive competencies of humans in general, rather than the unique behavioural tendencies of individuals. The working hypothesis is that by incorporating known competencies (and indeed limitations) of human cognition into the collaborative behaviours of robots, they would be able better serve their human partners, particularly in cases where interactions are short-term, and/or prior to adaptation on an individual level.

As such, this is proposed to be distinct from, but fully compatible and consistent with, existing approaches to the generation of collaborative robot behaviours. These include, for example, legible motion generation [2], and optimal task planning and allocation [4],

Permission to make digital or hard copies of part or all of this work for personal or classroom use is granted without fee provided that copies are not made or distributed for profit or commercial advantage and that copies bear this notice and the full citation on the first page. Copyrights for third-party components of this work must be honored

For all other uses, contact the owner/author(s).

HRI '18 Companion, March 5-8, 2018, Chicago, IL, USA

(c) 2018 Copyright held by the owner/author(s).

ACM ISBN 978-1-4503-5615-2/18/03.

https://doi.org/10.1145/3173386.3177070

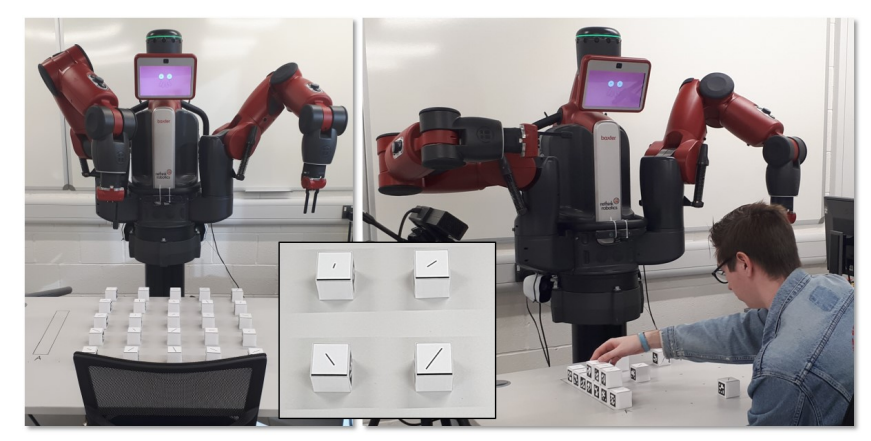

Figure 1: Baxter robot setup used: (left) arrangement of blocks prior to interaction, (right) a participant engaged in the sorting task, (inset) example stimuli used - lower right of inset is an example of a stimulus in category $B$, and upper left of inset an example of category $A$.

where the robots are typically intended to provide complementary physical expertise to that of the human, or assist the human by predicting their future task-relevant actions and providing prospective support as appropriate [5]. It is in this context that we suggest that collaborative robots could also take into account the limitations of the human at a more fundamental cognitive level.

\section{PROOF-OF-CONCEPT STUDY}

The study participants were asked to sort a set of blocks, arranged in a grid (figure 1), into one of two categories, based on the stimulus image appearing on each block. The role of the robot in this task was to provide suggestions to the participant regarding in which category each block should be placed. It did so by pointing to a block and verbally suggesting a target category. The incidence of suggestions was based on participant behaviour: if there was no categorisation activity for 5 seconds, the robot would make a suggestion. No explicit turn-taking mechanism was implemented or enforced, and the robot suggestions were always correct.

The stimuli set is based on stimuli used in psychological categorical perception experiments, e.g. [1,3]. Each stimulus is defined according to two dimensions (line length and line orientation, see figure 1 inset for examples). In this study, 25 stimuli were employed, and the two groups (of equal number of stimuli) were formed based on line length (short lines in category A, and long lines in category B), where line orientation was a distractor (i.e. did not predict category). One stimulus lay on the category boundary (and thus neither in category A nor B). The participants were not made aware of this category membership rule, they had to learn this through the interaction. 

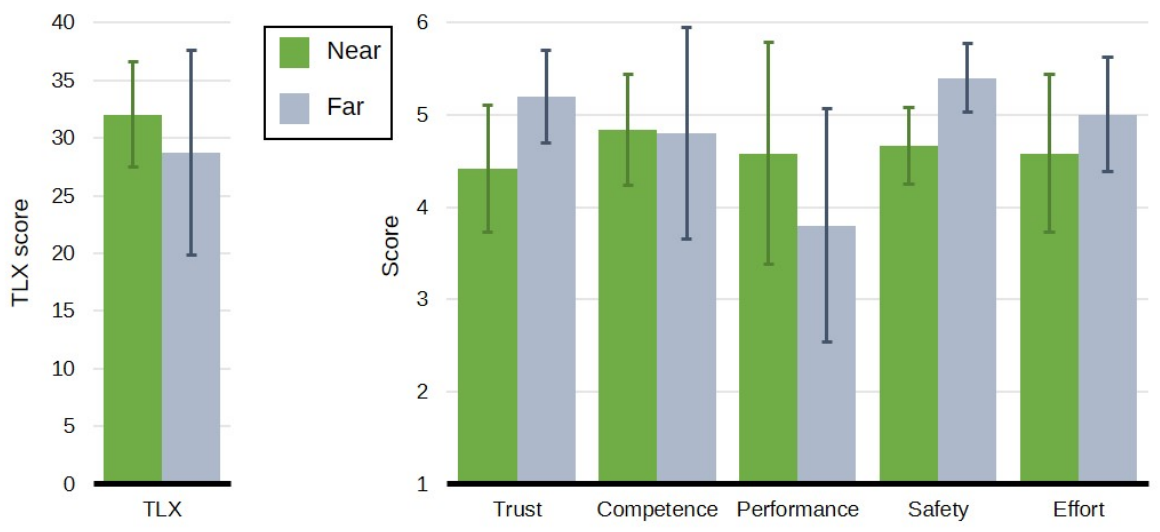

Figure 2: Questionnaire data for both a standard NASA TLX (left), and five subscales introduced in [6] related to subjective acceptance (right). Error bars show 95\% CI.

Each participant repeated the categorisation task four times, with the scene reset by the experimenter after each trial: all 25 stimuli were randomly arranged in a grid, although this randomly ordered initial configuration was consistent for all trials, for all participants. Participants were randomly assigned to one of two conditions: either the robot would first preferentially choose to highlight stimuli which lay close to the category boundary before moving to stimuli further away from the category boundary (Near condition), or first highlighting those stimuli far from the category boundary (Far condition). The procedure lasted around 40 minutes per participant (including questionnaires).

A total of 14 people participated, with 3 withdrawn due to system errors (student sample: age range 19-21, leaving 9 male, 2 female), in a between-subject design. The classification accuracy of participants was automatically logged, although the primary focus of this contribution is to examine participant impressions of the two conditions with respect to perceived task workload (NASA TLX), and perception of the robot (including trust, competence, performance, etc), as employed in [6]. These questionnaires were administered at the end of the four trials.

\section{RESULTS AND DISCUSSION}

Despite the context of a proof-of-concept study with only limited participants, the results indicate some trends that support further investigation. For example, an initial assumption underlying the two experimental conditions was that the Near condition - in which the robot would first suggest close-to-category-boundary stimuli - would be perceived as more helpful since the robot would be assisting with those stimuli that are predicted (from perceptual categorisation theory) to be more difficult to resolve.

Participant feedback however indicates that the reverse effect seems to have occurred: by identifying clear category members first in the Far condition, the classification rule appears to have been more readily discoverable. The metrics provide some support for this view (figure 2), although the small sample size reduces the utility of inferential statistics. For example, perceived workload (TLX) is higher and trust in the system is lower for the Near condition. Perceived safety is also higher for the Far condition. However, other results are not entirely consistent with this view: perceived performance is higher for the Near condition behaviour.

Further study is clearly required to provide further insight into these issues. What is nevertheless demonstrated is the effect of robot behaviour on human performance, and perception thereof, which is directly informed by fundamental human cognitive faculties. We do not suggest this as a replacement for adaptation to the individual, but rather a complementary mechanism that could lower initial barriers to interaction.

While in this paper we present only a preliminary illustration of the concept, it lays the foundation for further developments in cognitive assistance and collaboration in side-by-side working for humans and robots by explicitly taking into consideration the cognitive competencies of humans.

\section{ACKNOWLEDGMENTS}

This work was partially supported by the Research Investment Fund (RIF) of the University of Lincoln, and the EU H2020 project 732773 ILIAD.

\section{REFERENCES}

[1] Kathryn L. Carpenter, Andy J. Wills, Abdelmalek Benattayallah, and Fraser Milton. 2016. A Comparison of the neural correlates that underlie rule-based and information-integration category learning. Human Brain Mapping 37, 10 (2016), 3557-3574. https://doi.org/10.1002/hbm.23259

[2] Anca D Dragan and Kenton C T Lee. 2013. Legibility and Predictability of Robot Motion. In Human-Robot Interaction (HRI), 2013 8th ACM/IEEE International Conference on. IEEE Press, Tokyo, Japan. https://doi.org/10.1109/HRI.2013.6483603

[3] J Vincent Filoteo, Scott Lauritzen, and W Todd Maddox. 2010. Removing the frontal lobes: the effects of engaging executive functions on perceptual category learning. Psychological Science 21, 3 (2010), 415-23. https://doi.org/10.1177/ 0956797610362646

[4] Lars Johannsmeier and Sami Haddadin. 2016. A hierarchical human-robot interaction-planning framework for task allocation in collaborative industrial assembly processes. IEEE Robotics and Automation Letters 3766, c (2016), 1-1. https://doi.org/10.1109/LRA.2016.2535907

[5] Brian Scassellati and Katherine M. Tsui. 2015. Co-Robots: Humans and Robots Operating as Partners. In Handbook of Science and Technology Convergence. Springer International Publishing, 1-10. https://doi.org/10.1007/978-3-319-04033-2_27-1

[6] Astrid Weiss, Andreas Huber, Jürgen Minichberger, and Markus Ikeda. 2016. First Application of Robot Teaching in an Existing Industry 4.0 Environment: Does It Really Work? Societies 6, 3 (2016), 20. https://doi.org/10.3390/soc6030020 